\title{
Poliquistosis hepática hemorrágica sin poliquistosis renal, una condición de alto riesgo vital: Reporte de caso, abril 2015 Perú
}

Daniel Cárdenas Ruiz de Castilla ${ }^{a}$, Eliseo Chirinos Marroquín ${ }^{1,2, c}$, Marco Vergara Labrín ${ }^{1,2, b}$, Carlos Del Castillo Parodi²,c

\section{RESUMEN}

La poliquistosis hepática sin poliquistosis renal es una condición clínica infrecuente y más aún su complicación hemorrágica, con muy pocos casos reportados, siendo fatal sin tratamiento precoz.

Mujer, 40 años, consulta por dolor en hipocondrio derecho, una hora de evolución, punzante, intensidad moderada; náuseas.

En Emergencia: PA 130/80mmHg, FC 100 lpm, T 37 C, saturación 0299 \%, FR 22 rpm. Abdomen blando. Dolor en hipocondrio derecho. No visceromegalias. Punto cístico y signo de Murphy positivo. Laboratorio: Hematocrito 34 , hemoglobina $11,4 \mathrm{~g} / \mathrm{dL}$, leucocitos 12190 , amilasa $70 \mathrm{U} / \mathrm{L}$.

Ecografía Abdominal: Poliquistosis hepática con ruptura de quiste, líquido subcapsular y abundante libre en cavidad, sin lesiones en páncreas ni riñones.

Se realiza laparoscopía exploradora de emergencia, drenaje y aspiración de líquido más quistectomía parcial. Evolución favorable, cita con familiares directos para estudios complementarios.

Palabras clave: Hepatopatías; hígado poliquistico; laparoscopía (Fuente: DeCS BIREME).

\section{Hemorrhagic polycystic liver disease without the presence of polycystic kidney disease, a serious life-threatening condition: a case report. April 2015, Peru}

\section{ABSTRACT}

Polycystic liver disease without the presence of polycystic kidney disease is a rare clinical condition and its hemorrhagic complication is even rarer, with very few cases being reported. It can be life-threatening without a rapid intervention. Case report: A 40 year-old female who presented with moderate sharp right upper quadrant abdominal pain and nausea for one hour.

Emergency department: blood pressure $130 / 80 \mathrm{mmHg}$, heart rate 100 beats per minute, temperature $37^{\circ} \mathrm{C}, 02$ saturation $99 \%$, respiratory rate 22 breaths per minute. On examination, soft and relaxed abdomen, right upper quadrant abdominal pain, no visceromegaly, positive Murphy's sign and rebound sign.

Labs: hematocrit $34 \%$, hemoglobin $11.4 \mathrm{~g} / \mathrm{dL}$, white blood cells 12190 , amylase $70 \mathrm{U} / \mathrm{L}$.

Abdominal ultrasound: Polycystic liver disease with ruptured cyst, abundant free subcapsular fluid within cavity, without pancreas and kidneys injury.

Procedures: emergency exploratory laparoscopy, fluid drainage and aspiration, and partial cystectomy. Favorable evolution. Appointment with patient's direct relatives for further studies.

Keywords: Liver diseases; polycystic liver; laparoscopy (Source: MeSH NLM).

1. Universidad de San Martín de Porres, Facultad de Medicina Humana. Lima, Perú.

2. Delca Medical. Lima, Perú.

a. Cirugía General y Laparoscópica, Sanna, Clínica El Golf. Lima, Perú.

b. Médico Internista y de Emergencias.

c. Médico Cirujano, Especialista en Emergencias y Desastres. 


\section{INTRODUCCIÓN}

El dolor abdominal en hipocondrio derecho, es un motivo de consulta común en los servicios de emergencias; y aunque primero se piensa en las causas más frecuentes de dolor no traumático, también es importante tener en cuenta las patologías más raras, que el uso creciente y generalizado de los estudios de imágenes han llevado al aumento en su detección de manera incidental.

La enfermedad poliquística hepática aislada sin poliquistosis renal, es una patología poco común y su complicación hemorrágica más aún; con muy pocos casos reportados a nivel mundial, lo que nos lleva a tener especial cuidado en pacientes con dolor abdominal, en este contexto, por su alta mortalidad.

\section{CASO CLÍNICO}

Se presenta paciente sexo femenino, 40 años de edad, que a las 06:00 a.m. consulta con el servicio de Emergencias de Clínica SANNA El Golf del distrito de San Isidro, por dolor abdominal súbito, punzante, intensidad moderada, en hipocondrio derecho, irradiado a epigastrio y región lumbar homolateral, de una hora de evolución, que se inició mientras realizaba gimnasia. Naúseas, sin diarrea, disuria, ni sensación febril. Ingesta de comida grasa la noche anterior.

Antecedentes patológicos: niega alergia a medicamentos, obesidad grado I, hace 8 meses dolor similar de menor intensidad.
Nacida en área rural de Piura, donde vive hasta los 2 años. Padre y hermana mayor con quistes en hígado.

Fecha de última menstruación hace 2 semanas, ciclos dolorosos. 6 gestas, 6 partos.Ingresa al servicio de Emergencia con PA 130/80 mmHg, FC 100 lpm, FR 22 rpm, $\mathrm{T} 37^{\circ} \mathrm{C}$, SatO2 $99 \%$. El examen físico muestra abdomen globuloso, blando y distendido. Dolor a palpación superficial y profunda con predominio de hipocondrio derecho y pelvis. No visceromegalias, ruidos hidroaéreos disminuidos. Punto epigástrico, cístico y Murphy (+). Signo de rebote presente.

Laboratorio: Hto $34 \%$, Hb 11,4g/dL, GB 12,190/ul, NS $85 \%$, PCR 10,6mg/L, glucemia $118 \mathrm{mg} / \mathrm{dL}$, AST $17 \mathrm{U} / \mathrm{L}$, ALT $13 \mathrm{U} / \mathrm{L}$, amilasa $70 \mathrm{U} / \mathrm{L}$, fosfatasa alcalina $73 \mathrm{U} / \mathrm{L}$, bilirrubina total $0,47 \mathrm{gr} / \mathrm{dl}$, ELISA para hidatidosis: negativa (informe posterior al ingreso). Examen de orina: 1-4 leucocitos y 11-15 hematíes por campo.

Ecografía abdominal: Poliquistosis hepática, hematoma intrahepático con ruptura de quiste, líquido subcapsular y abundante libre en cavidad, litiasis vesicular múltiple, sin lesiones en páncreas ni ambos riñones.

Solicitamos TAC abdominal con contraste endovenoso, mostrando extensa ocupación del parénquima hepático por quistes de varios tamaños en ambos lóbulos (Clasificación Gigot III) (Figura 1).

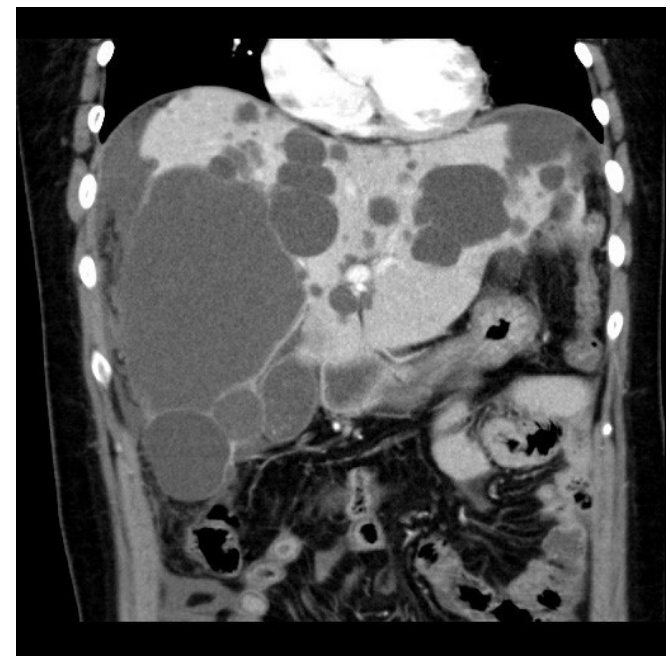

Figura 1. Tomografía abdominal, corte coronal, que evidencia el extenso compromiso del parénquima hepático

Se realiza laparoscopía exploradora de emergencia, se encontró gran compromiso quístico de ambos lóbulos hepáticos, además la ruptura de un quiste de $10 \mathrm{~cm}$ de diámetro en segmento VI y $1500 \mathrm{cc}$ de líquido serohemático libre en cavidad peritoneal; se procede a descapsulación del quiste (quistectomía parcial), para controlar sangrado por dentro del mismo, luego aspiración de líquido y lavado de abdomen. Se revisa toda la cavidad, dejando drenaje a circuito cerrado (Figura 2). 


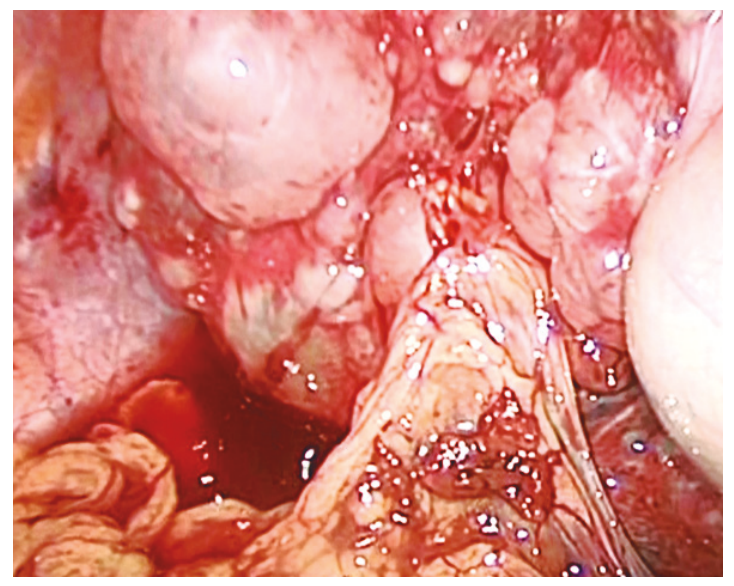

Figura 2. Cirugía laparoscópica que muestra el quiste hepático roto en el segmento VI

La anatomía patológica del material informa: pared fibrosa de quiste, tapizada por epitelio simple plano con infiltrado inflamatorio mixto y hemorragia focal.

La paciente permanece hospitalizada dos días, sin complicaciones inmediatas. Se realiza seguimiento evolutivo por consultorios externos, retirándose dren a la semana, e invitando a los familiares directos, para estudios complementarios.

\section{DISCUSIÓN}

El dolor abdominal localizado en hipocondrio derecho, es un motivo común de consulta en el servicio de emergencia.
Las características en cada forma de presentación reveladas con la confección de una detallada historia clínica nos permiten acercarnos al diagnóstico concreto de la patología, que confirmaremos con la ayuda de los exámenes auxiliares ${ }^{(1)}$.

Aunque hay que pensar primero en las causas más frecuentes de dolor abdominal no traumático del hipocondrio derecho, es importante tener en cuenta las patologías más raras, que el uso creciente y masivo de los estudios por imágenes ha llevado al aumento en su detección en forma incidental ${ }^{(2,3)}$.

Tabla 1. Antecedentes demográficos de población estudiada. Huechuraba, 2016

\begin{tabular}{ll} 
Colecistitis Aguda & \multicolumn{1}{c}{ Cólico biliar } \\
Colelitiasis & Colangitis \\
Hepatitis aguda & Apendicitis retrocecal \\
Úlcera duodenal & Neumonía basal derecha \\
Infarto de miocardio & Insuficiencia cardiaca congestiva \\
Herpes zoster & Pancreatitis aguda \\
Absceso hepático & Quiste hepático hidatídico \\
Cólico renal & Pielonefritis aguda \\
Obstrucción intestinal & Gastroenteritis \\
Vólvulo colónico & Isquemia mesentérica \\
Porfírias & Síndrome urémico \\
Cetoacidosis & Trauma abdominal \\
Intoxicación alimentaria & Intoxicación con plomo, alcohol
\end{tabular}


La poliquistosis hepática aislada, es decir, sin asociarse a poliquistosis renal autosómica dominante $(80 \%$ de los casos), es una enfermedad de presentación infrecuente y rara, desconociendo su real prevalencia, se calcula en $1 / 100,000$, e incidencia $<0,01 \%{ }^{(4,5)}$.

Se ha identificado una relación con el gen localizado en el cromosoma 19p, con un comportamiento genético distinto a la asociada con quistes renales, pero con una patogénesis y manifestaciones clínicas similares ${ }^{(6)}$. Más recientemente se ha comunicado determinadas mutaciones en el gen PRKCSH (Protein Kinase C Substrate $80 \mathrm{~K}-\mathrm{H}$ ), que codifica la proteína hepatocistinas, en el retículo endoplásmico del hepatocito, originando la poliquistosis hepática autosómica dominante ${ }^{(7)}$.

La poliquistosis hepática es una enfermedad con heterogeneidad clínica y genética; los quistes proceden de la dilatación de microhamartomas biliares y de glándulas peribilares que pueden identificarse en ecografía, TAC o RMN.

Esta patología hepática se presenta con mayor frecuencia en mujeres, y a una edad más temprana que los hombres (40 años aproximadamente), siendo más grave en multigestantes ${ }^{(8)}$. Cursa habitualmente en forma asintomática (80\%), si produce manifestaciones clínicas son por efecto de masa, con distensión abdominal, sensación de plenitud, reflujo gastroesofágico, dispepsia, disnea o dolor lumbar, o bien por complicaciones agudas (hemorragia, infección o ruptura). Las pruebas de laboratorio suelen ser normales ${ }^{(9)}$.

La poliquistosis hepática aislada puede presentarse con un fenotipo de afectación vascular hasta en el 5,6 \%, con aneurismas cerebrales y anomalías valvulares cardiacas, como prolapso de válvula mitral ${ }^{(10)}$.

La ecografía (como gold estándar) y la TAC, resultan útiles como técnicas no invasivas para establecer el diagnóstico diferencial y seguimiento de las lesiones. El hígado se caracteriza por presencia difusa de múltiples quistes, que ocupan más del $50 \%$ del parénquima, en número menor a diez en forma de grandes masas $(>10 \mathrm{~cm})$ o en medianos y pequeños nódulos, clasificación de Gigot ${ }^{(11)}$. En los pacientes que pertenecen a una familia afectada, los criterios diagnósticos incluyen más de un quiste en $<40$ años y más de tres en > 40 años; sin familiares afectados, el criterio habitual es presentar más de 20 quistes ${ }^{(12)}$.

El diagnóstico diferencial incluye quistes hepáticos múltiples asociados a enfermedad renal poliquística autosómica dominante, enfermedad de Caroli (quistes en comunicación con las vías biliares que se diagnostica usando contrastes de excreción biliar), hamartoma de conductos biliares, hemangioma cavernoso, neoplasia quística primaria, cistoadenoma, metástasis quística, cistoadenocarcinoma, absceso, hidatidosis, hematoma y bilioma intrahepático.

Un importante problema de salud pública, por su alta frecuencia como parasitosis hepática, son los quistes de Echinococcus granulosus, con 9,3 \% de prevalencia asintomática en áreas endémicas del Perú ${ }^{(13)}$. Aunque no se cuenta con datos actualizados, ni exactos; la presentación de quistes únicos es la consigna más frecuente, a diferencia de los casos de quistes múltiples $(18,4 \%)^{(14)}$.

Recientemente el lantreótido y el octreótido (análogos de somatostatina) han demostrado ser seguros y efectivos en la reducción volumétrica del hígado poliquístico y también previniendo su crecimiento al impedir la acumulación de líquido por reducción del AMPC intracelular ${ }^{(15-17)}$, pero requieren cumplimiento de por vida. Cuando los síntomas son severos, existen terapéuticas quirúrgicas, como la aspiración percutánea y esclerosis de quistes, que tienden a preservar la mayor cantidad posible de tejido hepático con disminución absoluta del dolor y tolerancia normal de la dieta ${ }^{(18,19)}$, u opciones más agresivas como hepatectomía parcial ${ }^{(20)}$. En cualquiera de los casos las soluciones son paliativas, no curativas; sin embargo, el trasplante hepático se ha convertido en el tratamiento de elección de la enfermedad hepática terminal, siendo la única opción curativa ${ }^{(21)}$.

El sangrado del quiste hepático es una complicación muy poco frecuente, una última revisión describe al décimo segundo caso reportado en la literatura; con mortalidad de $25 \%$, uno solo de poliquístosis hepática sin quistes renales y un solo paciente con manejo laparoscópico ${ }^{(22)}$.

En conclusión, la enfermedad poliquística hepática aislada es una patología poco frecuente y su complicación hemorrágica más aún, pero nos lleva a tener especial cuidado en pacientes con dolor abdominal, en este contexto, por su alta mortalidad.

Agradecimiento: A la Sra. Sylvia Wong Lau, por sus continuos aportes y colaboración en la realización de este trabajo.

\section{REFERENCIAS BIBLIOGRÁFICAS}

1. Chanana L, Jegaraj M, Kalyaniwala K, Yadav B, Abilash K. Clinical profile of non-traumatic acute abdominal pain presenting to an adult emergency department. J Family Med Prim Care. 2015;4(3):422-5. Disponible en: https://www. ncbi.nlm.nih.gov/pmc/articles/PMC4535107/

2. Marrero J, Ahn J, Rajender K. American College of Gastroenterology. Clinical guideline: the diagnosis and management of focal liver lesions. Am J Gastroenterol. 2014;109(9):1328-47. Disponible en: https://www.nature. com/articles/ajg2014213

3. Peces R, González P, Venegas J. Enfermedad poliquistica hepatica no asociada a poliquistosis renal autosomica dominante. Nefrología. 2003;23(5):454-458. Disponible en: http://www.revistanefrologia.com/es-publicacionnefrologia-articulo-enfermedad-poliquisticahepatica-no-asociada-poliquistosis-renal-autosomicadominante-X0211699503016241

4. Russell R, Pinson C. Surgical management of polycystic liver disease. World J Gastroenterol. 2007;13(38):50529. Disponible en: https://www.ncbi.nlm.nih.gov/pmc/ 
articles/PMC4434633/

5. Patel A, Shah R. Polycystic liver disease presenting as acute abdomen. QJM. 2014;107(9):769. Disponible en: https:// www.ncbi.nlm.nih.gov/pubmed/24453280

6. Janssen $M$, Waanders $E$, Te Morsche R, Xing R, Dijkman H, Woudenberg J, et al. Secondary, somatic mutations might promote cyst formation in patients with autosomal dominant polycystic liver disease. Gastroenterology. 2011;141(6):205663. Disponible en: https://www.ncbi.nlm.nih.gov/ pubmed/21856269

7. Banales J, Munoz-Garrido P, Bujanda L. Somatic secondhit mutations leads to polycystic liver diseases. World J Gastroenterol. 2013;19(1):141-3. Disponible en: https:// www.ncbi.nlm.nih.gov/pmc/articles/PMC3542753/

8. Van Keimpema L, De Koning D, Van Hoek B, Van Den Berg A, Van Oijen M, De Man R, et al. Patients with isolated polycystic liver disease referred to liver centres: clinical characterization 137 cases. Liver Int. 2011;31(1):92-8. Disponible en: https:// www.ncbi.nlm.nih.gov/pubmed/20408955

9. Hogan M, Abebe K, Torres V, Chapman A, Bae K, Tao C, et al. Liver involvement in early autosomal-dominant polycystic kidney disease. Clin Gastroenterol Hepatol. 2015;13(1):15564. Disponible en: https://www.ncbi.nlm.nih.gov/ pubmed/25111236

10. Gevers T, de Koning D, Van Dijk A, Drenth J. Low prevalence of cardiac valve abnormalities in patients with autosomal dominant polycystic liver disease. Liver Int. 2012;32(4):6902. Disponible en: https://www.ncbi.nlm.nih.gov/ pubmed/22099398

11. Gigot J, Jadoul P, Que F, Van Beers B, Etienne J, Horsmans Y, et al. Adult polycystic liver disease: is fenestration the most adequate operation for long-term management?. Ann Surg. 1997;225(3):286-94. Disponible en: https://www.ncbi.nlm. nih.gov/pmc/articles/PMC1190679/

12. Abu-Wasel B, Walsh C, Keough V, Molinari M. Pathophysiology, epidemiology, classification and treatment options for polycystic liver diseases. World J Gastroenterol. 2013;19(35):5775-86. Disponible en: https://www.ncbi.nlm. nih.gov/pmc/articles/PMC3793132/

13. Grosso G, Gruttadauria S, Biondi A, Marventano S, Mistretta A. Worldwide epidemiology of liver hydatidosis including the Mediterranean area. World J Gastroenterol. 2012;18(13):142537. Disponible en: https://www.ncbi.nlm.nih.gov/pmc/ articles/PMC3319938/

14. García P, Chabez A, Fernández M, Guerra M, Levano I, Quiroz S, et al. Características clínicas y epidemiológicas de hidatidosis en el Hospital Nacional Dos de Mayo. Revista de Medicina Humana-Universidad R Palma. 2006;6(2):26-30. Disponible en: http://aulavirtual1.urp.edu.pe/ojs/index.php/RFMH/ article/viewFile/23/19

15. Temmerman F, Dobbels F, Ho TA, Pirson $Y$, Vanslembrouck R, Coudyzer W, et al. Development and validation of a polycystic liver disease complaint-specific assessment (POLCA). J Hepatol. 2014;61(5):1143-50. Disponible en: http://www. sciencedirect.com/science/article/pii/S0168827814004541

16. Spirli C, Okolicsanyi S, Fiorotto R, Fabris L, Cadamuro M, Lecchi S, et al. ERK1/2-dependent vascular endothelial growth factor signaling sustains cyst growth in polycystin-2 defective mice. Gastroenterology. 2010;138(1):360-71. Disponible en: https://www.ncbi.nlm.nih.gov/pubmed/19766642

17. Temmerman F, Vanslembrouck R, Coudyzer W, Bammens B, Laleman W, Cassiman D, et al. The reduction in liver volume in polycystic liver disease with lanreotide is dose dependent and is most pronounced in patients with the highest liver volume. J. Hepatol. 2012;56(2):S547.Disponible en: https://www. infona.pl/resource/bwmeta1.element.elsevier-7e1a775bdd99-3813-9557-1873c893ad76

18. Duarte-Chang C. Avances en el manejo de la Poliquistosis Hepática. Rev méd cient. 2012;25(2):26-31. Disponible en: http://www.revistamedicocientifica.org/uploads/ journals/1/articles/367/public/367-1556-1-PB.pdf

19. Ampuero J, Bañales J, Soriano G, Crespo J, Olcoz J, Diago $M$, et al. La poliquistosis hepática del adulto (PHA) en España: análisis de una encuesta estructurada analizando la experiencia y actitud de los especialistas de digestivo españoles. Rev Esp Enferm Dig. 2014;106(4):263-75. Disponible en: http://scielo.isciii.es/scielo.php?pid=S1130$01082014000400005 \&$ script=sci_arttext\&tlng=en

20. Dietrich A, Ardiles V, Lendoire J, Raffin G, Moro M, Storck G, et al. Liver resection for non tumoral liver disease. Multicentric experience in Argentina. Actas Gastroenterol Latinoam. 2014;44(2):114-20. Disponible en: http://europepmc.org/ abstract/med/25199305

21. Tseng J, Orloff SL. Management of symptomatic polycystic liver disease with hepatic resection. JAMA Surg. 2015;150(1):812. Disponible en: https://jamanetwork.com/journals/ jamasurgery/fullarticle/1934726

22. Marion Y, Brevart C, Plard L, Chiche L. Hemorrhagic liver cyst rupture: an unusual life-threatening complication of hepatic cyst and literatura review. Ann. Hepatol 2013;12(2):336-9. Disponible en: http://www.medigraphic.com/pdfs/hepato/ ah-2013/ah132w.pdf

\section{Fuentes de Financiamiento:}

Este artículo ha sido financiado por los autores.

\section{Conflictos de interés:}

Los autores declaran no tener ningún conflicto de interés.

\section{Correspondencia:}

Sylvia Wong Lau

Dirección: Calle Quito Mz. J1 Lote 8. Urb. Sta. Patricia, La Molina. Teléfono: 946524191

Correo electrónico: sylviawonglau@hotmail.com

Recibido: 28 de febrero de 2017 Evaluado: 01 de marzo de 2017 Aprobado: 31 de marzo de 2017

(c) La revista. Publicado por Universidad de San Martín de Porres, Perú. (c) в 1 Licencia de Creative Commons Artículo en acceso abierto bajo términos de Licencia Creative Commons Atribución 4.0 Internacional. (http://creativecommons.org/licenses/by/4.0/)

\section{ORCID iDs}

Daniel Cardenas Ruiz de Castilla Marco Antonio Vergara Labrin Carlos Del Castillo Parodi Eliseo Chirinos https: / / orcid.org/0000-0003-0504-630X https://orcid.org/0000 000212068550 https://orcid.org/0000 00018583 276X https://orcid.org/0000 000325308880 